\title{
Review of: "Open Innovation in Response to Covid 19 :A case study of the Open Source Ventilator Ireland Project"
}

\author{
Roberto Rivas Hermann ${ }^{1}$
}

1 Nord University

Potential competing interests: The author(s) declared that no potential competing interests exist.

Dear author.

It was a very interesting paper to read and learn about the application of open innovation strategies in the context of the global pandemic caused by the COVID-19 disease. The paper presents an in depth-case study of the development of an open-source ventilator and the network attached to this initiative, involving partners throughout the world. Besides the practical implications of the paper, it also develops a modified perspective of Chesbrough's open innovation funnel framework, grounded on the case study.

Considering this as a preprint version which aims to rapidly share the results with the overall community in the pandemic, the format of the article is not necessarily that of a conventional journal manuscript. As result, I read this more as a rapid or field communication, which of course requires a reframing of its structure vis-à-vis a potential journal submission. If that is the purpose, there are several aspects to improve.

First, is the theoretical framing which seems to be hidden behind the contextual description of the case study, clearly rooted in the story behind the development of the ventilator right from the start. This somehow hidden the state of the art behind open innovation, and more in concrete communities/ platforms for open innovation. What is the current state of this debate? How your case adds to that debate, and what is the theoretical contribution? Maybe the contribution is connected to debates within the bio-medical industry which is also valid. However this is not mentioned from the start I think.

Second, is the lack of an analytical framework to understand the case. You do a good effort by the end by introducing the model of open innovation, and connecting key concepts in this figure (spin-offs, open innovation, etc). However, these are important concepts to clarify right from the start. Perhaps one source that could be useful here is the paper by (Garud, Tuertscher, and Van de Ven 2013), or similar references which provide a better framing of the different levels as part of the innovation process (community, organization, etc...).

Third, methodologically the article reads as a narrative, and as an account based on primarily and secondary data. Yet, this needs to be framed in a methodology section. Which currently lacks in the manuscript.

To finalize, I would like to congratulate the author by the interesting conclusion which opens up some reframing of the open innovation model and is grounded in a rich case. My only suggestion here, will be to make this more explicit about how this differs from other research which has analyze open innovation in other contexts, including conflicts, or platforms. 


\section{Reference}

Garud, Raghu, Philipp Tuertscher, and Andrew H. Van de Ven. 2013. "Perspectives on Innovation Processes." The Academy of Management Annals 7 (1): 775-819. https://doi.org/10.1080/19416520.2013.791066. 